\title{
Localization and expression of histone H2A variants during mouse oogenesis and preimplantation embryo development
}

\author{
B.J. Wu ${ }^{1,2 *}$, F.L. Dong ${ }^{1,3 *}$, X.S. Ma ${ }^{1}$, X.G. Wang ${ }^{1}$, F. Lin ${ }^{1}$ and H.L. Liu ${ }^{1}$ \\ ${ }^{1}$ College of Animal Science and Technology, Nanjing Agricultural University, \\ Nanjing, China \\ ${ }^{2}$ Inner Mongolia Saikexing Reproductive Biotechnology Co., Ltd., \\ Helingeer, China \\ ${ }^{3}$ Institutes of Biology and Medical Sciences, Soochow University, \\ Suzhou, China \\ *These authors contributed equally to this study. \\ Corresponding author: H.L. Liu \\ E-mail: liuhonglin@263.net
}

Genet. Mol. Res. 13 (3): 5929-5939 (2014)

Received September 13, 2013

Accepted February 20, 2014

Published August 7, 2014

DOI http://dx.doi.org/10.4238/2014.August.7.8

\begin{abstract}
Epigenetic modifications of the genome, such as histone $\mathrm{H} 2 \mathrm{~A}$ variants, ensure appropriate gene activation or silencing during oogenesis and preimplantation embryo development. We examined global localization and expression of the histone $\mathrm{H} 2 \mathrm{~A}$ variants, including H2A.Bbd, H2A.Z and H2A.X, during mouse oogenesis and preimplantation embryo development. Immunocytochemistry with specific antibodies against various histone $\mathrm{H} 2 \mathrm{~A}$ variants showed their localization and changes during oogenesis and preimplantation development. H2A.Bbd and H2A.Z were almost absent from nuclei of growing oocytes (except 5-day oocyte), whereas H2A.X was deposited in nuclei throughout oogenesis and in preimplantation embryos. In germinal vesicle (GV) oocyte chromatin, H2A.Bbd was detected as a weak signal, whereas no fluorescent signal was detected in GV
\end{abstract}


breakdown (GVBD) or metaphase II (MII) oocytes; H2A.Z showed intense signals in chromatin of GV, GVBD and MII oocytes. H2A. Bbd showed very weak signals in both pronucleus and 2-cell embryo nuclei, but intense signals were detected in nuclei from 4-cell embryo to blastula. The H2A.Z signal was absent from pronucleus to morula chromatin, whereas a fluorescent signal was detected in blastula nuclei. Our results suggest that histone $\mathrm{H} 2 \mathrm{~A}$ variants are probably involved in reprogramming of genomes during oocyte meiosis or after fertilization.

Key words: H2A variants; Preimplantation embryo; Oogenesis; Epigenetics; Reprogramming

\section{INTRODUCTION}

In most eukaryotes, histones are major structural components of chromatin, and various core histones have many different variants. Different histone variants can contribute to a distinct or unique nucleosomal architecture, where this heterogeneity can be exploited to regulate a wide range of nuclear functions, and evidence is accumulating that histone variants do indeed have distinct functions.

Among the core histones, H2A has the largest number of variants, including H2A.Z, MacroH2A, H2A-Bbd, and H2A.X (Kamakaka and Biggins, 2005). Some H2A variants, such as H2A.Z, are conserved through evolution (Zlatanova and Thakar, 2008); while others such as MacroH2A and H2A-Bbd (Eirín-López et al., 2008) are restricted to vertebrates or mammals. The $\mathrm{H} 2 \mathrm{~A}$ variants are distinguished from the major $\mathrm{H} 2 \mathrm{~A}$ histones by their $\mathrm{C}$-terminal tails, which diverge in both length and sequence, as well as in their genome distribution. MacroH2A localizes predominantly to the inactive X chromosome (Mietton et al., 2009), while H2ABbd localizes to the active $X$ chromosome and autosomes (Eirín-López et al., 2008). H2A.X and $\mathrm{H} 2 \mathrm{~A} . \mathrm{Z}$ are constitutively expressed and localize throughout the genome, although H2A.Z shows some enrichment in intergenic regions. In Drosophila, a single variant called H2AvD has sequence characteristics of both H2A.X and H2A.Z (Redon et al., 2002).

In mammals, H2A.Z is encoded by an essential gene, since homozygous knockout of the gene in mice results in embryos that fail to develop beyond gastrulation (Faast et al., 2001). H2A.Z is usually enriched at the transcriptional start sites of genes and plays critical roles in gene regulation through transcriptional activation (Bonisch and Hake, 2012), and the acetylation of H2A.Z shows a strong correlation with gene deregulation and epigenetic remodeling (Valdes-Mora et al., 2012). The C-terminus of H2A.Z contains a short region that is essential for development beyond the larval stages in Drosophila melanogaster (Clarkson et al., 1999). In Tetrahymena thermophila, H2A.Z was found exclusively in the transcriptionally active macronucleus and was expressed in the silent macronucleus only during conjugation before gene activation. Previously, H2A.Z was shown to localize to pericentric heterochromatin in early mouse development, and knockdown of H2A.Z levels by RNAi in mammalian cell lines led to chromosome missegregation and disruption of the normal distribution of the heterochromatin-specific protein HP1 $\alpha$ (Rangasamy et al., 2004).

H2A.X is a histone variant in higher eukaryotes, which, although absent in nematodes, is the normal histone H2A in budding yeast (Downs et al., 2000). The yeast H2A and the 
higher eukaryote H2A.X histones contain an extension at the C-terminus, which includes the conserved amino acid sequence SQ (E/D) $\varphi$ (where $\varphi$ denotes a hydrophobic residue). Ser139 in this unique C-terminal region is phosphorylated in response to DNA double-strand breaks (DSBs) and seems to be an early step in the response to DNA damage. The deletion of H2A.X in mice, although not lethal, causes a reduction in the number of irradiation-induced foci and results in genomic instability and male infertility (Celeste et al., 2002).

MacroH2A is a vertebrate-specific variant, which has two distinct domains: the Nterminus, which is similar to that of $\mathrm{H} 2 \mathrm{~A}$, and a large $\mathrm{C}$-terminus, which has no similarity to that of the other histone H2A variants. MacroH2A is enriched on the inactive X (Xi) chromosome in mammalian female cells (Mietton et al., 2009). Although this variant is a hallmark of $\mathrm{X}$ inactivation, its presence is not essential for maintenance of the inactivated state. Its deposition occurs after localization of the inactive X-specific transcript, Xist, on the Xi chromosome (Splinter et al., 2011). In the absence of the Xist transcript, macroH2A cannot localize to the $\mathrm{Xi}$ chromosome. This suggests that Xist RNA may be involved in promoting histone exchange. MacroH2A has a general role in silencing, as evidenced by findings that the Cterminal 'macro' domain inhibits the binding of transcription factors (Angelov et al., 2003).

$\mathrm{H} 2 \mathrm{~A}$. Bbd is the most recently isolated H2A variant and little is known about it, with the following exceptions. It is excluded from the Xi chromosome in mammalian cells and colocalizes with acetylated H4/K12 (Kim et al., 2003) and can regulate transcription and mRNA processing in human cells (Tolstorukov et al., 2012). Interestingly, Gautier et al. (2004) showed that the mobility of H2A.Bbd in the nucleus is faster than that of H2A. A recent study (Ishibashi et al., 2010) reported that the native form of H2A.Bbd is present in highly advanced spermatogenic fractions of mammalian testis at the time when histones are highly acetylated and being replaced by protamines. It is also present in the nucleosomal chromatin fraction of mature human sperm.

In this study, we characterized chromatin incorporation and deposition of histone $\mathrm{H} 2 \mathrm{~A}$ variants H2A.Bbd, H2A.Z and H2A.X during mouse oogenesis and early embryo development. We found that H2A.X was abundantly incorporated and deposited into chromatin in mouse oocytes and early embryos. However, H2A.Bbd and H2A.Z, minimal or no incorporation of these proteins was observed during oogenesis and the preimplantation stage after fertilization. These results suggest that global changes in chromatin structure, associated with altered histone composition, are involved in genome remodeling after fertilization.

\section{MATERIAL AND METHODS}

\section{Ethics statement}

Animal studies were carried out in an SPF animal facility accredited by the Association for the Assessment and Accreditation of Laboratory Animal Care, and all animal protocols were approved by the Animal Care and Use Committee of the Model Animal Research Center, the host for the National Resource Center for Mutant Mice in China, Nanjing University.

\section{Chemicals}

Unless otherwise indicated, all chemicals were purchased from Sigma (St. Louis, MO, USA). Pregnant mare serum gonadotropin (PMSG) and human chorionic gonadotropin (hCG) 
were purchased from Ningbo Sansheng pharmaceutical Co., Ltd. (China).

\section{Animals and recovery of oocytes and embryos}

Ovaries from 1-, 5-, 10- and 15-day-old Kunming female mice were harvested and washed in phosphate-buffered saline (PBS) three times, then digested in $100 \mu \mathrm{L} 0.25 \%$ trypsinEDTA (Invitrogen, Carlsbad, CA, USA) to collect oocytes at various stages. Ovaries from 1-and 5-day-old mice were incubated at $37^{\circ} \mathrm{C}$ for $5 \mathrm{~min}$, followed by mixing for $5 \mathrm{~min}$, and 1 - and 5 -day mouse oocytes were collected. Ovaries from 10- and 15-day-old mice were digested with $0.25 \%$ trypsin-EDTA, followed by mixing for 10-15 min, and collected 10- and 15-day mouse oocytes.

Kunming female mice at 21-28 days old were superovulated with 10 IU PMSG and then treated $48 \mathrm{~h}$ later with $10 \mathrm{IU}$ hCG. Germinal vesicle (GV) oocytes were collected from ovaries harvested from Kunming mice after PMSG $48 \mathrm{~h}$ later. GV oocytes were incubated in a humidified atmosphere of $5 \% \mathrm{CO}_{2}$ and $95 \%$ air at $37^{\circ} \mathrm{C}$ in $\mathrm{KSOM}$ medium for $4-6 \mathrm{~h}$ to collect GV breakdown (GVBD) oocytes. Unfertilized metaphase II-arrested (MII) oocytes were collected 14-15 h later in Whitten's medium from the ampullae of the oviducts.

Kunming female mice at 21-28 days old were superovulated to provide embryos as previously described (Nagy, 2003). After superovulation with 10 IU PMSG followed by 10 IU hCG $48 \mathrm{~h}$ later, females were paired with Kunming males and inspected the following morning for copulation plugs. Preimplantation embryos were flushed from the oviducts after the hCG treatment. Pronuclear stage embryos (2PN) were harvested and fixed at $22 \mathrm{~h}, 2$-cell embryos at $48 \mathrm{~h}, 4$-cell embryos at $60 \mathrm{~h}, 8$-cell and 9- to 12-cell embryos at $70 \mathrm{~h}$, morula embryos at $84 \mathrm{~h}$, and blastocyst embryos at $104 \mathrm{~h}$, after hCG injection.

\section{Immunofluorescence and fluorescence microscope}

Oocytes and embryos were prepared for immunofluorescence by fixation in a 3.7\% paraformaldehyde solution at room temperature for $1 \mathrm{~h}$. They were then washed in PBS with $0.2 \%$ Tween-20 three times, and left in blocking buffer (PBS/0.2\% Tween-20 and $10 \mathrm{mg} / \mathrm{mL}$ $\mathrm{BSA}$ ) for $1 \mathrm{~h}$ at $37^{\circ} \mathrm{C}$. Oocytes and embryos were then double-stained to visualize various histone $\mathrm{H} 2 \mathrm{~A}$ variants and DNA. Briefly, samples were incubated in anti-histone $\mathrm{H} 2 \mathrm{~A}$ variant antibody (Abcam, UK) diluted 1:200-1:100 for $1 \mathrm{~h}$ at $37^{\circ} \mathrm{C}$ or overnight at $4^{\circ} \mathrm{C}$. Oocytes and embryos were then washed in $\mathrm{PBS} / 0.2 \%$ Tween-20 three times and incubated in a Cy3or FITC-conjugated anti-mouse or rabbit IgG secondary antibody (1:200, Abcam) for $1 \mathrm{~h}$ at $37^{\circ} \mathrm{C}$. Finally, oocytes or embryos were washed three times in PBS $/ 0.2 \%$ Tween-20, stained for DNA with $3 \mathrm{~g} / \mathrm{L}$ DAPI for $20 \mathrm{~min}$, and mounted in PBS containing 50\% glycerol and 25 $\mathrm{mg} / \mathrm{mL} \mathrm{NaN}_{3}$. Oocytes and embryos were analyzed with a fluorescence microscope.

Reagents (Abcam) used for immunofluorescence analyses were as follows. Primary antihistone H2A variant antibodies: H2A.Z antibody-ChIP Grade (ab4174, Abcam), H2A.X antibody (ab20669, Abcam, UK), and H2A-Bbd antibody (ab4175, Abcam). Secondary antibodies: antirabbit IgG antibody-H\&L (ab6939, Abcam) and anti-mouse IgG antibody-H\&L (ab6724, Abcam).

\section{Semiquantitative RT-PCR analysis}

Total RNA was extracted from mouse oocytes or embryos using Trizol LS reagent (Invi- 
trogen) according to manufacturer recommendations. Extracted RNA was stored in $75 \%$ ethanol at $-80^{\circ} \mathrm{C}$ until used. RNA was resuspended in $10 \mu \mathrm{L}$ nuclease-free water and used in the assay. First-strand complementary DNA (cDNA) was synthesized using Moloney murine leukemia virus reverse transcriptase (MMLV RT) (Promega, Madison, WI, USA) in a $25-\mu \mathrm{L}$ final reaction volume. Briefly, $5 \mu \mathrm{L}$ RNA was incubated with $1 \mu \mathrm{L}$ random primers (50 ng $\mu \mathrm{L}$ ) (Promega) and $4 \mu \mathrm{L}$ nuclease-free water in a $10-\mu \mathrm{L}$ final reaction volume at $70^{\circ} \mathrm{C}$ for $5 \mathrm{~min}$ and then cooled on ice to accomplish nucleic acid denaturing. After incubation on ice, $4.5 \mu \mathrm{L}$ nuclease-free water, $5 \mu \mathrm{L} 5 \mathrm{X}$ reaction buffer ( $250 \mathrm{mM}$ Tris- $\mathrm{HCl}, \mathrm{pH} 8.3$, at $25^{\circ} \mathrm{C}, 375 \mathrm{mM} \mathrm{KCl}, 15 \mathrm{mM} \mathrm{MgCl}{ }_{2}, 50 \mathrm{mM}$ DTT), $1 \mu \mathrm{L} 10 \mathrm{mM}$ of each deoxynucleoside triphosphate, $0.5 \mu \mathrm{L} 40 \mathrm{U} / \mu \mathrm{L}$ RNA in ribonuclease inhibitor (Promega) and $1 \mu \mathrm{L} 200 \mathrm{U} / \mu \mathrm{L}$ MMLV RT were added, and the reaction mixture was further incubated at $42^{\circ} \mathrm{C}$ for $60 \mathrm{~min}$. RT-PCR was performed in a single tube using the same $2 \mathrm{X}$ Taq PCR Master Mix (Tiangen Biotech, Beijing, China). The reaction mixture consisted of $12.5 \mu \mathrm{L}$ Master Mix, $1 \mu \mathrm{L}$ forward primer, $1 \mu \mathrm{L}$ reverse primer (see Table 1), 4-6 $\mu \mathrm{L} \mathrm{cDNA}$ and $\mathrm{ddH}_{2} \mathrm{O}$ to $25 \mu \mathrm{L}$. The RT-PCR thermal cycling program consisted of $95^{\circ} \mathrm{C}$ for $5 \mathrm{~min}$, followed by 35 cycles of $94^{\circ} \mathrm{C}$ for $30 \mathrm{~s}, 45^{\circ}-65^{\circ} \mathrm{C}$ (see Table 1) for $30 \mathrm{~s}$ and $72^{\circ} \mathrm{C}$ for $1 \mathrm{~min}$, followed by a 5 -min extension cycle of $72^{\circ} \mathrm{C}$. Amplified samples were then electrophoresed on a $2 \%$ agarose gel for $30-45 \mathrm{~min}$. Following ethidium bromide staining, bands were visualized and relative intensities determined using the Kodak Digital Science TM1D software (Eastman Kodak Co., Rochester, NY, USA).

Table 1. Primer sequences used for PCR amplification.
\begin{tabular}{lllc}
\hline \\
Primer & Forward sequence & Reverse sequence & Tm \\
\hline H2A.X & 5'-ACCACCTCCCTCACAGAA-3' & 5'-GACACCGAAGGAAACAATG-3' & $60.8^{\circ} \mathrm{C}$ \\
H2A.Z & 5'-CAGGAAATGCGTCAAAAGA-3' & 5'-CCACTGGAATCACCAACAC-3' & $60.8^{\circ} \mathrm{C}$ \\
$\beta$-actin & $5^{\prime}$-GCTGTCCCTGTATGCCTCT-3' & 5'-GTCTTTACGGATGTCAACG-3' & $60.8^{\circ} \mathrm{C}$ \\
\hline
\end{tabular}

\section{RESULTS}

\section{Expression of histone variants during mouse oogenesis and in preimplantation embryos}

To investigate the dynamic deposition of the histone $\mathrm{H} 2 \mathrm{~A}$ variants during mouse oogenesis and in preimplantation embryos, we determined the mRNA expression levels of H2A.Z and H2A.X using semiquantitative RT-PCR. We detected the signal of mRNA in all three periods, which included oogenesis, oocyte meiosis and preimplantation embryo (Figure 1A and B). We also found that H2A.Bbd could be detected in the oocyte cytoplasm using the method of paraffin section immunocytochemistry (Figure 1C). We then continued to examine the changes in histone variant incorporation sites in the three periods using immunocytochemistry.

\section{Localization of histone $\mathrm{H} 2 \mathrm{~A}$ variants during mouse oogenesis}

First, the histone H2A variants were examined during mouse oogenesis. Oocytes at various stages of growth were evaluated by immunocytochemistry with specific antibodies against various histone H2A variants (Figure 2). H2A.Bbd signal was absent from 1-, 10- and 15-day oocyte chromatin. By contrast, H2A.Bbd signal increased in the cytoplasm. These findings indicated that H2A.Bbd localized in the cytoplasm (Figure 1C and Figure 2A) during the growth of 
the oocytes. Immunocytochemistry revealed that H2A.Bbd was deposited in only 5-day oocytes around the nuclear area, which suggested that H2A.Bbd was assembled from the cytoplasm to localize around the nuclear area in the 5-day oocyte. In the 20-day oocyte, the same H2A.Bbd signal was detected in the nucleus and cytoplasm (Figure 2A); H2A.Z signal was also absent from 1-, 5-, 10- and 15-day oocyte chromatin. By contrast, H2A.Z signal increased in the cytoplasm. These data suggest that H2A.Z may function in the cytoplasm of early growing oocytes. In the 20day oocyte, H2A.Z signal started to be deposited in the nucleus (Figure 2B); an abundant signal for H2A.X persisted in 1-, 10-, 15- and 20-day oocyte chromatin. Thus, all of the H2A.X signal localized in growing oocyte chromatin, except for 5-day oocytes. The H2A.X signal present in 5-day oocytes was around the nuclear area (Figure 2C). Histone $\mathrm{H} 2 \mathrm{~A}$ variants did not localize in growing oocyte chromatin, except for H2A.X. However, histone H2A variant mRNAs were present in the cytoplasm (Figure 1A and B). This suggested that some transfer of histone H2A variant protein was inhibited in growing oocytes. Our findings indicated that histone H2A variants are incorporated during oogenesis and have different localization in the oocyte.

A
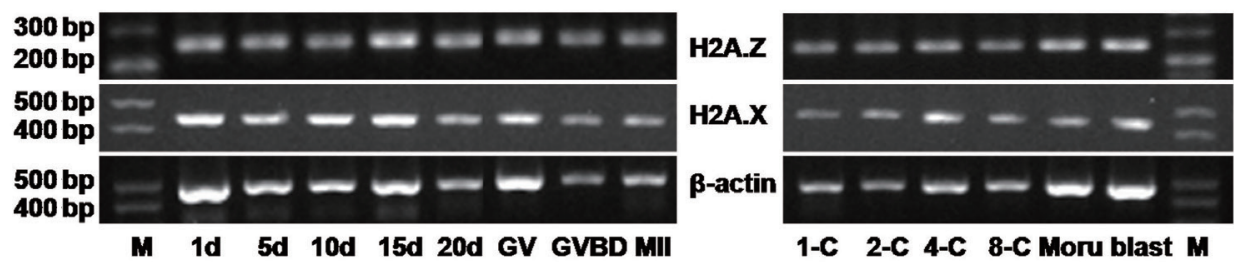

300 bp $200 \mathrm{bp}$

$500 \mathrm{bp}$ 400 bp

$500 \mathrm{bp}$ $400 \mathrm{bp}$

B

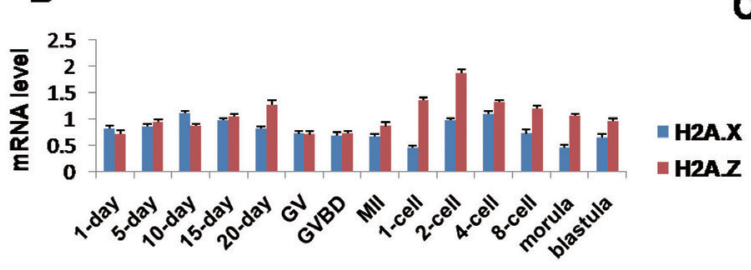

C

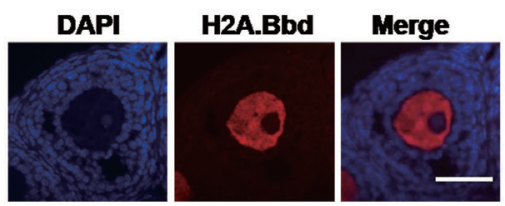

Figure 1. Expression levels of histone variants during mouse oogenesis and in preimplantation embryos. A. Semiquantitative RT-PCR analysis: mRNA levels of histone H2A variants during oogenesis and in preimplantation embryos. Histone H2A variant cDNA was amplified by semiquantitative PCR. PCR products were separated on a $2 \%$ agarose gel in which $\beta$-actin cDNA served as an internal control. Lane $M=$ DNA marker. B. A graph was plotted using the quantitative measurements of each amplified product using the Image-J program (mRNA level $=$ target gene/ $\beta$-actin). Bar graphs represent means \pm SE. C. Mouse follicle paraffin section was subjected to immunocytochemistry with anti-H2A.Bbd antibody. The antibody was localized with Cy3-conjugated secondary antibodies (red), and DNA was stained with DAPI (blue). H2A.Bbd signal was detected in oocyte cytoplasm, but not in the oocyte nucleus. Scale bars: $50 \mu \mathrm{m}$.

\section{Histone H2A variants incorporated in oocyte meiosis}

During oocyte meiosis, GV, GVBD and MII oocytes were analyzed by immunocytochemistry with specific antibodies against various histone H2A variants (Figure 3). Our experimental results indicated that H2A.Bbd was deposited in GV oocyte chromatin (Figure 3A), 


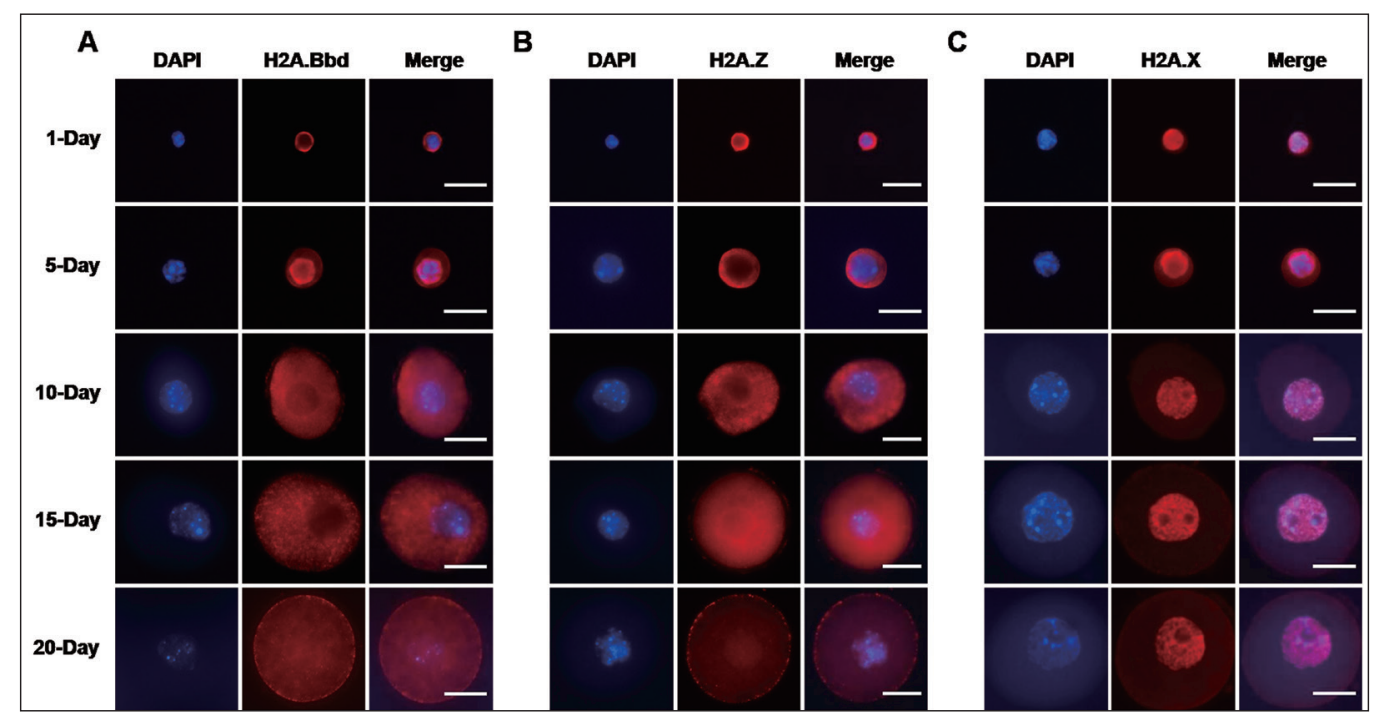

Figure 2. Localization and expression of histone $\mathrm{H} 2 \mathrm{~A}$ variants during mouse oogenesis. Oocytes at various growth stages: 1-, 5-, 10-, 15- and 20-day oocytes were subjected to immunocytochemistry with the various anti-histone H2A variant antibodies. A. B. and C., respectively, indicate H2A.Bbd, H2A.Z and H2A.X protein in 1-, 5-, 10-, 15- and 20-day oocytes. The antibody was localized with Cy3-conjugated secondary antibodies (red), and DNA was stained with DAPI (blue). Scale bars: $50 \mu \mathrm{m}$.

whereas H2A.Bbd signals were absent from GVBD and MII oocyte chromatin. It suggests that H2A.Bbd is possibly involved in the oocyte reprogramming of gene expression. H2A.Z was present as intense signals in chromatin of GV, GVBD and MII oocytes (Figure 3B), and H2A.X signals were detected throughout oocyte meiosis (Figure 3C). The results showed that H2A.X was possibly important for the maintenance of genomic stability in mice. Although, in GVBD and MII stage oocytes, some H2A variants were not detected in chromatin, but mRNA was present in the cytoplasm (Figure 1A and B).

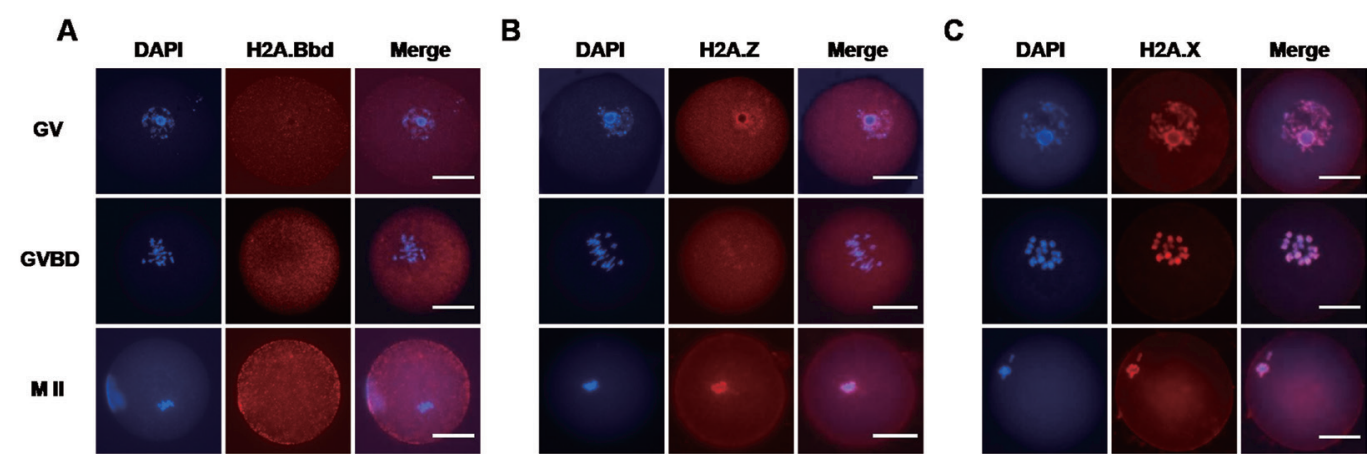

Figure 3. Localization and expression of histone H2A variant in GV, GVBD and MII stage oocytes. GV, GVBD and MII stage oocytes were subjected to immunocytochemistry with the various anti-histone H2A variant antibodies. A. B. and C., respectively, indicate H2A.Bbd, H2A.Z and H2A.X protein fluorescent signals. The antibody was localized with Cy3-conjugated secondary antibodies (red), and DNA was stained with DAPI (blue). Scale bars: $50 \mu \mathrm{m}$. 


\section{Localization of histone $\mathrm{H} 2 \mathrm{~A}$ variants in preimplantation embryos}

Histone $\mathrm{H} 2 \mathrm{~A}$ variants were examined in preimplantation embryos. Immunocytochemistry with specific antibodies against various histone $\mathrm{H} 2 \mathrm{~A}$ variants showed fluorescence signals for histone $\mathrm{H} 2 \mathrm{~A}$ variants in preimplantation embryos. In our assays, a very weak signal for H2A.Bbd was present in 1-cell and 2-cell embryo chromatin. Especially, almost no H2A.Bbd signals were detected in the 1-cell embryo. Intense H2A.Bbd signals were detected from the 4-cell embryo to blastula stage (Figure 4A). It suggests that H2A.Bbd is possibly involved in the propagation of genomic function information from one cell generation to the next by retaining this information during mitosis and is possibly involved in the reprogramming of gene expression. Fluorescent signals for H2A.Z were not detected from pronucleus to morula, whereas abundant signals were found at the blastula stage (Figure 4B). An intense signal for H2A.X persisted in mouse preimplantation embryo chromatin (Figure 4C). As described above, cytoplasm dramatically changed after fertilization. This change may have been caused by de novo protein synthesis after fertilization (Fuchimoto et al., 2001). These newly synthesized proteins could change the properties of the embryonic cytoplasm dramatically. Thus, histone H2A variants were incorporated in mice during early embryo development, and there was histone H2A variant mRNA expression in preimplantation embryos (Figure 1A and B).
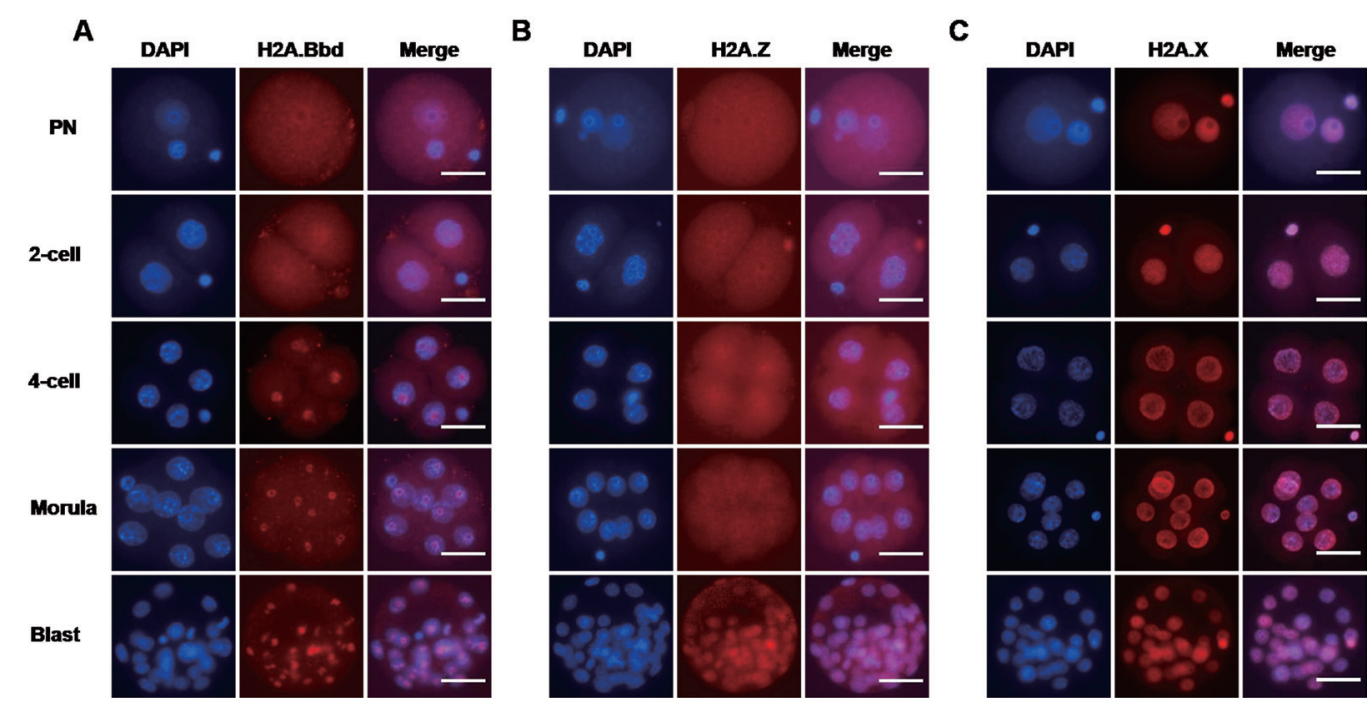

Figure 4. Localization and expression of histone H2A variants in preimplantation embryos. Preimplantation embryos at various stages were subjected to immunocytochemistry with the various anti-histone H2A variant antibodies. A. B. and C., respectively, indicate H2A.Bbd, H2A.Z and H2A.X fluorescent signals. The antibody was localized with Cy3-conjugated secondary antibodies (red), and DNA was stained with DAPI (blue). Scale bars: $50 \mu \mathrm{m}$.

\section{DISCUSSION}

One of the interesting aspects of epigenetics is the role of histone variants, which are used by the cell to build specialized nucleosomes by replacing canonical histones at different 
stages of the cell cycle and generating an architecturally and functionally distinct chromatin structure (Ausió and Abbott, 2002). The histone H2A family encompasses the greatest diversity of variants among core histones, which can substitute canonical replication-dependent H2A (Sarma and Reinberg, 2005). The incorporation of these particular histone variants into nucleosomes has significant impacts on transcriptional activation, DNA repair, meiosis, and apoptosis (Kamakaka and Biggins, 2005).

In our study, mRNA levels of histone $\mathrm{H} 2 \mathrm{~A}$ variants varied in growing oocytes and during oocyte meiosis (Figure 1A and B). During oogenesis, histone H2A variants did not accumulate in oocyte chromatin, but were present in the cytoplasm (Figure 2). To examine whether H2A.Bbd accumulated in the cytoplasm, we used the paraffin section immunocytochemistry method. This result suggested that H2A.Bbd truly accumulated in the cytoplasm (Figure 1C). After fertilization, most histone $\mathrm{H} 2 \mathrm{~A}$ variants were transferred to nuclear chromatin (Figure 4). It is possible that the amount of these variant proteins stored in oocytes changes after fertilization, leading to changes in the level of nuclear deposition. This change may be caused by de novo protein synthesis after fertilization (Fuchimoto et al., 2001). These newly synthesized proteins could change the properties of the embryonic cytoplasm dramatically. These data suggested that fertilization causes transfering the histone $\mathrm{H} 2 \mathrm{~A}$ variant proteins to nucleus. Minor activation of the embryonic genome occurs in the male pronucleus of the 1-cell embryo, but the two major waves of activation are observed first at the 2-cell stage and then at the 4- to 8-cell transition (Li et al., 2010).

Our results showed that $\mathrm{H} 2 \mathrm{~A}$.Bbd decreased dramatically during oocyte meiosis (Figure $3 \mathrm{~A}$ ), which suggests that $\mathrm{H} 2 \mathrm{~A} . \mathrm{Bbd}$ is involved in the reprogramming of gene expression. We also demonstrated that $\mathrm{H} 2 \mathrm{~A}$.Bbd was incorporated during mitosis in preimplantation embryos (Figure 4A). These results suggest that H2A.Bbd is involved in the propagation of genomic function information from one cell generation to the next by retaining this information during mitosis. Although gene expression patterns are inherited by the next generation in differentiated somatic cells, the gene expression patterns of immature oocytes, which are also differentiated cells, need to be reprogrammed, and the new program is established before or after fertilization. This reprogramming of gene expression probably involves global epigenetic modifications. When differentiated cells proliferate, the information on the active gene is transmitted faithfully to the daughter cell, thereby maintaining the lineage-specific program of gene expression in the descendent generations. As most of the transcription factors are displaced from the chromosomes and since the genes are silent during mitosis (Martínez-Balbás et al., 1995), it has been suggested that a mechanism exists to maintain the lineage-specific program of gene expression. This mechanism is called "cell memory" (Cavalli, 2006), and it suggests that certain epigenetic modifications remain as markers of cell memory in the chromosomes during mitosis (Turner, 1998). In this study, H2A.Bbd may have represented a marker of cell memory, where it decreased dramatically in oocytes during meiosis (Figure 3A) and was generally present in preimplantation embryos (Figure 4A).

H2A.Z is highly conserved across species and is essential for the viability of various organisms (Zlatanova and Thakar, 2008). In many organisms, H2A.Z is essential for survival; however, it is not important for early embryonic development. In mice, H2A.Z-deficient embryos develop normally until implantation (Faast et al., 2001). In our study, we found that H2A.Z was re-expressed at detectable levels starting at the blastocyst stage when differentiation takes place (Nashun et al., 2010) (Figure 3B). Recently, Creyghton et al. (2008) demonstrated 
that H2A.Z is not required for the maintenance of undifferentiated embryonic stem cells, but is important for lineage commitment during differentiation. Thus, we suggest that H2A.Z is reexpressed in embryos when it is required to fulfill a specific function related to differentiation.

Higher-order chromatin structure presents a barrier to the recognition and repair of DNA damage. DSBs induce histone H2A.X phosphorylation, which is associated with the recruitment of repair factors to damaged DNA. Thus, H2A.X is critical for facilitating the assembly of specific DNA repair complexes on damaged DNA. Nevertheless, loss of H2A.X leads to increased chromosomal abnormalities, deficiencies in gene targeting, and radiation sensitivity. Our results showed that H2A.X was incorporated during oocyte growth and preimplantation embryo development (Figures $2 \mathrm{C}, 3 \mathrm{C}$ and $4 \mathrm{C}$ ). Thus, histone H2A.X is very important for the maintenance of genomic stability in mice (Celeste et al., 2002). In undifferentiated mouse embryonic stem cells, H2A.X is abundant and its level decreases with differentiation (Shechter et al., 2009). Taken together with our findings that H2A.X is also present at high levels in early preimplantation embryos, abundant H2A.X could be involved in the maintenance of proliferating and undifferentiated states in higher eukaryotes.

A recent study showed that mammalian SWI/SNF complexes contribute to DSB repair by direct mechanisms of stimulating the phosphorylation of histone H2A.X $(\gamma-\mathrm{H} 2 \mathrm{~A}$.X) at DSB-surrounding chromatin. $\gamma$-H2A.X recruit SWI/SNF chromatin remodeling complexes (Park et al., 2009). In our study, at 1-cell embryo showed a very intense signal for H2A.X$\mathrm{S}_{139}$, whereas a very weak signal was detected in the 2-cell embryo. From 4-cell embryo to morula, intense H2A.X-S ${ }_{139}$ fluorescence signals were detected, whereas at blastula stage, the H2A.X-S ${ }_{139}$ fluorescence signal disappeared gradually (data not shown). This suggested that in mouse preimplantation embryos H2A.X-S ${ }_{139}$ may recruit SWI/SNF chromatin remodeling complexes and play an important role in the ATP-dependent chromatin remodeling process. Interestingly, the incorporation of H2A.X and H2A.X-S ${ }_{139}$ in mouse preimplantation embryos differs. H2A.X is incorporated during preimplantation embryo development (Figure $4 \mathrm{C}$ ) and its functions are not clear (data not shown).

In conclusion, histone $\mathrm{H} 2 \mathrm{~A}$ variants are thought to play an important role in oocyte reprogramming during meiosis to allow the initiation of a new program by totipotent zygotes of the next generation. Notably, chromatin localization of histone H2A variants changes dynamically during preimplantation development, and the formation of a unique chromatin structure as a result of the active incorporation and elimination of particular variants seems to facilitate the reprogramming event.

\section{ACKNOWLEDGMENTS}

Research supported by the Key Project of Chinese National Programs for Fundamental Research and Development (“973” Program \#2007CB947403).

\section{REFERENCES}

Angelov D, Molla A, Perche PY, Hans F, et al. (2003). The histone variant macroH2A interferes with transcription factor binding and SWI/SNF nucleosome remodeling. Mol. Cell 11: 1033-1041.

Ausió J and Abbott DW (2002). The many tales of a tail: carboxyl-terminal tail heterogeneity specializes histone H2A variants for defined chromatin function. Biochemistry 41: 5945-5949.

Bonisch C and Hake SB (2012). Histone H2A variants in nucleosomes and chromatin: more or less stable? Nucleic Acids 
Res. 40: 10719-10741.

Cavalli G (2006). Chromatin and epigenetics in development: blending cellular memory with cell fate plasticity. Development 133: 2089-2094.

Celeste A, Petersen S, Romanienko PJ, Fernandez-Capetillo O, et al. (2002). Genomic instability in mice lacking histone H2AX. Science 296: 922-927.

Clarkson MJ, Wells JR, Gibson F, Saint R, et al. (1999). Regions of variant histone His2AvD required for Drosophila development. Nature 399: 694-697.

Creyghton MP, Markoulaki S, Levine SS, Hanna J, et al. (2008). H2AZ is enriched at polycomb complex target genes in ES cells and is necessary for lineage commitment. Cell 135: 649-661.

Downs JA, Lowndes NF and Jackson SP (2000). A role for Saccharomyces cerevisiae histone H2A in DNA repair. Nature 408: 1001-1004.

Eirín-López JM, Ishibashi T and Ausio J (2008). H2A.Bbd: a quickly evolving hypervariable mammalian histone that destabilizes nucleosomes in an acetylation-independent way. FASEB J. 22: 316-326.

Faast R, Thonglairoam V, Schulz TC, Beall J, et al. (2001). Histone variant H2A.Z is required for early mammalian development. Curr. Biol. 11: 1183-1187.

Fuchimoto D, Mizukoshi A, Schultz RM, Sakai S, et al. (2001). Posttranscriptional regulation of cyclin A1 and cyclin A2 during mouse oocyte meiotic maturation and preimplantation development. Biol. Reprod. 65: 986-993.

Gautier T, Abbott DW, Molla A, Verdel A, et al. (2004). Histone variant H2ABbd confers lower stability to the nucleosome. EMBO Rep. 5: 715-720.

Ishibashi T, Li A, Eirin-Lopez JM, Zhao M, et al. (2010). H2A.Bbd: an X-chromosome-encoded histone involved in mammalian spermiogenesis. Nucleic Acids Res. 38: 1780-1789.

Kamakaka RT and Biggins S (2005). Histone variants: deviants? Genes Dev. 19: 295-310.

Kim JM, Liu H, Tazaki M, Nagata M, et al. (2003). Changes in histone acetylation during mouse oocyte meiosis. J. Cell Biol. 162: 37-46.

Li L, Zheng P and Dean J (2010). Maternal control of early mouse development. Development 137: 859-870.

Martínez-Balbás MA, Dey A, Rabindran SK, Ozato K, et al. (1995). Displacement of sequence-specific transcription factors from mitotic chromatin. Cell 83: 29-38.

Mietton F, Sengupta AK, Molla A, Picchi G, et al. (2009). Weak but uniform enrichment of the histone variant macroH2A1 along the inactive X chromosome. Mol. Cell Biol. 29: 150-156.

Nagy A (2003). Manipulating the Mouse Embryo: A Laboratory Manual. Cold Spring Harbor Laboratory Press, Cold Spring Harbor.

Nashun B, Yukawa M, Liu H, Akiyama T, et al. (2010). Changes in the nuclear deposition of histone H2A variants during pre-implantation development in mice. Development 137: 3785-3794.

Park JH, Park EJ, Hur SK, Kim S, et al. (2009). Mammalian SWI/SNF chromatin remodeling complexes are required to prevent apoptosis after DNA damage. DNA Repair 8: 29-39.

Rangasamy D, Greaves I and Tremethick DJ (2004). RNA interference demonstrates a novel role for H2A.Z in chromosome segregation. Nat. Struct. Mol. Biol. 11: 650-655.

Redon C, Pilch D, Rogakou E, Sedelnikova O, et al. (2002). Histone H2A variants H2AX and H2AZ. Curr. Opin. Genet. Dev. 12: 162-169.

Sarma K and Reinberg D (2005). Histone variants meet their match. Nat. Rev. Mol. Cell Biol. 6: 139-149.

Shechter D, Chitta RK, Xiao A, Shabanowitz J, et al. (2009). A distinct H2A.X isoform is enriched in Xenopus laevis eggs and early embryos and is phosphorylated in the absence of a checkpoint. Proc. Natl. Acad. Sci. U. S. A. 106: 749-754.

Splinter E, de Wit E, Nora EP, Klous P, et al. (2011). The inactive X chromosome adopts a unique three-dimensional conformation that is dependent on Xist RNA. Genes Dev. 25: 1371-1383.

Tolstorukov MY, Goldman JA, Gilbert C, Ogryzko V, et al. (2012). Histone variant H2A.Bbd is associated with active transcription and mRNA processing in human cells. Mol. Cell 47: 596-607.

Turner BM (1998). Histone acetylation as an epigenetic determinant of long-term transcriptional competence. Cell Mol. Life Sci. 54: 21-31.

Valdes-Mora F, Song JZ, Statham AL, Strbenac D, et al. (2012). Acetylation of H2A.Z is a key epigenetic modification associated with gene deregulation and epigenetic remodeling in cancer. Genome Res. 22: 307-321.

Zlatanova J and Thakar A (2008). H2A.Z: view from the top. Structure 16: 166-179. 